\title{
Numerical Computations of Fluid Flow and Heat Transfer in Gas Injected Iron Baths
}

\author{
Hasmet TURKOGLU and Bakhtier FAROUK
}

Mechanical Engineering and Mechanics Department, Drexel University, Philadelphia, PA 19104, U.S.A.

(Received on February 26, 1990; accepted in the final form on June 29, 1990)

The time evolution of the flow and temperature fields in industrial scale gas-injected molten iron baths are numerically analyzed. For the vertical injection case, a cylindrical vessel, containing molten iron, is considered. For the horizontal injection system, a cubical vessel is chosen. The Eulerian approach is used for the formulation of both the gas and the liquid phase transports. Turbulence in the liquid phase is predicted using a two-equation $k-\varepsilon$ Model. A constant effective viscosity is used for the gas phase turbulence. For the interphase friction and heat transfer coefficients, correlations from the literature are used. In order to realistically model the volume expansion due to gas injection, the computational domain is extended beyond the initial undisturbed liquid height.

KEY WORDS: mathematical modeling; ladle metallurgy; steelmaking; mixing

\section{Introduction}

Gas injection continues to play an increasingly important role in metallurgical processing operations. Some examples, where gas injection is widely used, are degassing of molten steel by injection of argon, desulfurization of a steel bath by powder injection using inert carrier gases, and inert gas stirring of molten metal baths to achieve homogeneous temperature and composition. In such systems gas bubbles rising through the liquid enhance mixing, promote chemical reactions, and minimize temperature and chemical inhomogeneities in the melt. The effectiveness of the gas injection in promoting these processes depends on the hydrodynamic behavior of the gas and the liquid phases, and their interaction with each other. Thus, a detailed analysis of the problem requires prediction of flow and temperature fields in the gas and melt.

A comprehensive experimental and numerical research project is underway in our laboratories to investigate the transport processes in gas injected liquid baths. The objectives are to obtain design guidelines for the novel concepts in steelmaking. The experiments are limited to bench-scale air-water and nitrogen-Wood's metal systems. A numerical model has been developed in parallel with the experimental study. Numerical model was also tested using experimental data reported by other investigators. A comparison of our numerical predictions with the measurements of Castillejos and Brimacombe ${ }^{1)}$ for an air-water system was reported earlier. ${ }^{2)}$ The experimental part of the research program consists of volume fraction and temperature measurements in various gas injection systems. In the present work, vertical and horizontal gas injection in industrial scale molten iron baths were considered.

In this paper, the computational results of gas in- jection processes in molten iron baths are presented. The flow, temperature and volume fraction fields were predicted for both the gas and the liquid phases over the whole domain. The problem was formulated on the basis of a two-fluid model using the Eulerian approach for both the gas and liquid phase transports. Turbulence in the liquid phase was predicted using the standard two-equation $k-\varepsilon$ turbulence model. Since gas phase turbulence has minor effect on the overall mixing process, a constant effective viscosity was assumed for the gas phase. The effects of the turbulence on the phase dispersion was included in the calculations by introducing a dispersion term in the mass continuity equation of the gas phase. For the vertical injection case, a cylindrical vessel was considered, shown in Fig. 1. For the horizontal injection system, a cubical vessel containing molten iron was selected. The geometry and the coordinate system of the problem are given in Fig. 2. In order to realistically model the volume expansion due to gas injection, the computational domain was extended beyond the initial undisturbed liquid height $H_{i}$ (see Figs. 1 and 2).

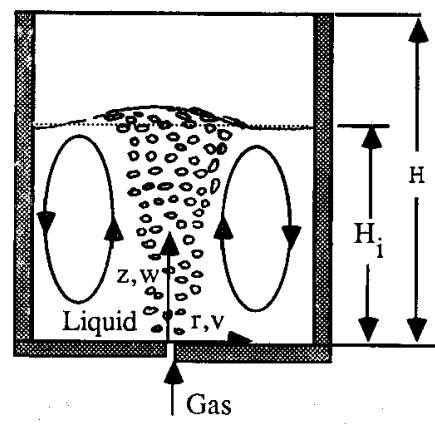

Fig. 1. Geometry and coordinate system of the problem considered for the vertical injection analysis. 


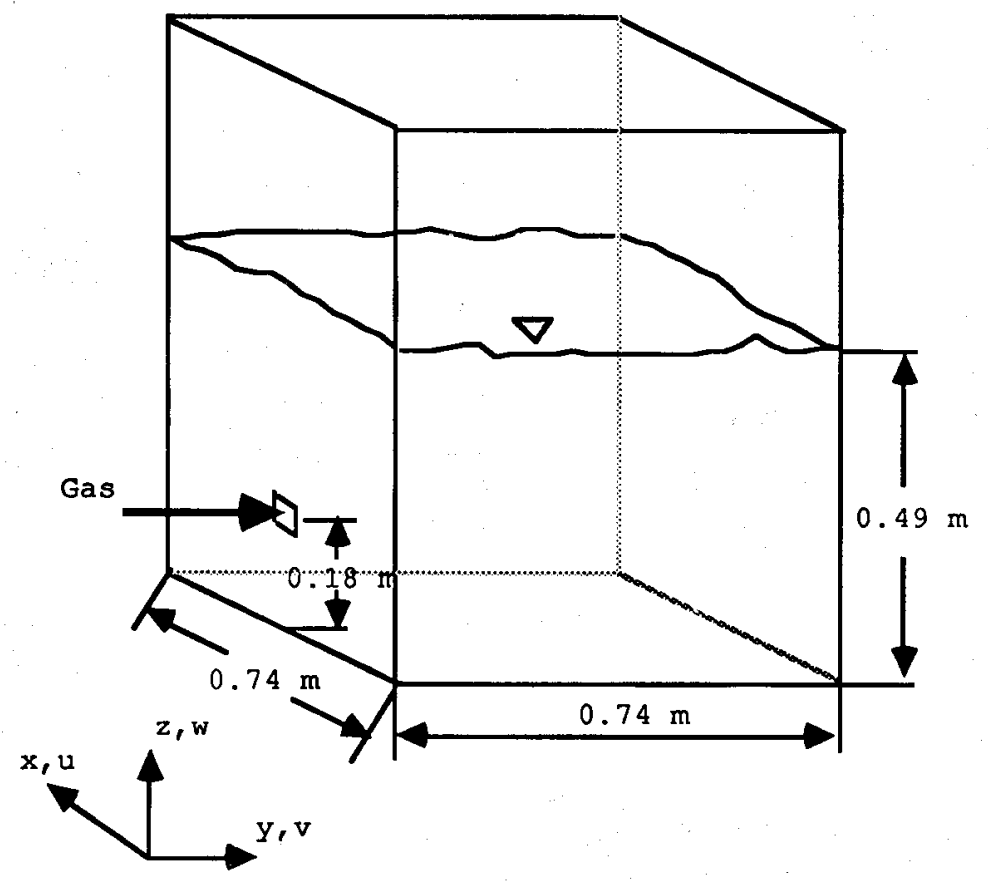

Fig. 2.

Geometry and coordinate systems of the horizontal injection systcm.

\section{Background}

In the injection systems, it is the coupling between the gas and the liquid phases that is responsible for creating a motion in the bulk of the liquid. The bubbles forming in the liquid rise upward due to buoyancy and the kinetic energy at the nozzle exit. The liquid experiences a force that is equal but opposite to the forces on the bubbles. Therefore, the liquid is accelerated and moves with the gas. The liquid further away from the two phase gas-liquid plume undergoes a circulation and agitation. The bulk motion of the liquid carries heat, momentum and dispersed materials along the streamlines, while eddy diffusivity transports those properties across the streamlines. These two factors, namely stream-wise bulk motion and eddy diffusivity, have the main roles in promoting mixing in the process vessel. Thus, the structure of the two-phase region, its interaction with the bulk of the liquid and recirculating turbulent motion outside the two-phase plume region should be studied to analyze the phenomena in a gas injected liquid bath.

In recent years, a considerable body of theoretical and experimental work concerning gas injection systems has been undertaken. ${ }^{1-12}$ In most of these studies, air and water were used to model the gas injection in metallurgical processes. In many of numerical studies, ${ }^{3-10)}$ it was assumed that gas penetration is confined in a cylindrical or conical region around the axis of the injection nozzle. The boundaries of this gas liquid plume region were determined from visual observations. The governing equations were only solved for the liquid phase with spatially varying density. The density of the mixture was calculated as a function of the gas volume fraction, while it was assumed to be equal to the liquid density outside the two-phase mixture region. The modeling used in all of the above mentioned studies is applica- ble for vertical injection systems only, as the dimensions of the gas-liquid plume region are not easy to determine for other systems. Other investigations ${ }^{13,14}$ have revealed that different gas-liquid systems lead to different behavior in the gas-liquid plume region. The above studies provided qualitative information on the hydrodynamic behavior of the liquid in a gas-injected bath. However, they require experimentally measured (geometric) data for the plume shape.

McKelliget et al. ${ }^{15)}$ investigated vertical (upward and downward) and horizontal injection cases in liquid baths. In this study, the gas-liquid mixture was treated as a continuous fluid of variable density. Dispersion of the gas was described by a turbulent diffusion equation. This approach predicted the vertical injection problems fairly well, however, it failed to predict the horizontal injection problems. Cross and Markatos ${ }^{16)}$ studied the vertical upward and/or downward injection (axi-symmetric and non-axisymmetric) into cylindrical vessels. In this study, they demonstrated the applicability of the multi-dimensional multiphase models for the analysis of such systems, however no detailed results were presented. Reccntly, Aldham et al., ${ }^{17)}$ Cross and Markatos, ${ }^{18)}$ Koh et al. ${ }^{19)}$ and Turkoglu and Farouk ${ }^{2)}$ used a twophase flow model to predict the flow fields in the gas and liquid phases. Air and water were used as the gas and liquid phases. The formulation used in these studies does not require any experimental information or assumption on gas plume boundary. Using the similar modeling approach, Schwarz et al. ${ }^{20)}$ analyzed the vertical nitrogen injection in a molten iron bath.

\section{Mathematical Formulation}

The gas-driven systems represent a two-phase turbulent recirculating flow problem. There is relative motion between the phases together with the interchange of momentum, heat and mass. Thus, the 
equations governing the phenomena should reflect these features of the problem. A complete analysis of the problem requires solution of the mass conservation, momentum and energy equations over the flow domain with appropriate boundary conditions for each phase.

The mathematical model used in the present study is based on a two-fluid (Eulerian-Eulerian) model. ${ }^{21)}$ The gas and liquid phases were considered to be two different continuous fields interpenetrating and interacting with each other. Mass conservation, momentum and energy equations were derived for each phase considering the time averaged variables. The phases exchange flow properties through the finite interphase areas. It was assumed that the pressure is the same in both phases within a computational cell. The field equations for each phase were weighted with the volume fraction of that phase. The exchanges between the phases were represented by source terms in conservation equations. The dispersion of phases due to turbulence was represented by introducing a diffusion term in the mass conservation equation of the gas phase, from which the phase volume fraction distribution was solved. The phase dispersion coefficient was calculated from turbulent viscosity of the liquid phase by introducing a dispersion Prandtl number.

\subsection{Governing Equations}

The governing equations are represented in vector form. The equations were however cast in the axisymmetric cylindrical coordinates $(r \times z)$ for the vertical case and in the Cartesian coordinates $(x, y, z)$ for the horizontal injection.

\subsubsection{Mass Conservation Equation}

In two-phase flows, one phase disperses into the other due to eddies at the interfaces of the phases and the concentration differences from one point to the other in the flow domain. This phenomena was formulated by introducing a dispersion term into the mass conservation equation of the gas phase, which was solved for the void fraction distribution. The mass conservation equation for each phase (in the absence of evaporation/condensation) is then given as:

$$
\frac{\partial}{\partial t}\left(R_{i} \rho_{i}\right)+\operatorname{div}\left(R_{i} \rho_{i} \vec{V}\right)-\operatorname{div}\left(\rho_{i} D_{t} \operatorname{grad} R_{i}\right)=0
$$

where, $R:$ the phase volume fraction

the subscript $i$ : the phase under consideration. The last term, which is zero for the liquid phase, represents the phase dispersion due to the turbulence. $D_{t}$ is the phase dispersion coefficient which was calculated as

$$
D_{t}=\frac{\nu_{t}}{\sigma_{d}}
$$

where, $\sigma_{d}:$ the dispersion Prandtl number

$\nu_{i}$ : the turbulence viscosity of the liquid phase.

For both the vertical and the horizontal injection cases, a dispersion Prandtl number of 1 was used.

\subsubsection{Momentum Equation}

The momentum equation for each phase is given as:

$$
\begin{aligned}
& \frac{\partial}{\partial t}\left(R_{i} \rho_{i} u_{i l}\right)+\operatorname{div}\left(R_{i} \rho \vec{V} u_{i l}\right) \\
& \quad=-\vec{l} \cdot R_{i} \operatorname{grad} P+B_{i l}+\operatorname{div}\left(R_{i} \mu_{\mathrm{eff}, i} \operatorname{grad} u_{i l}\right)+I_{i l}
\end{aligned}
$$

where, $B_{i l}$ : the $l$-direction body force per unit volume

$u_{i l}$ : the velocity of phase ' $i$ ' in direction ' $l$ '. The third term on the right-hand side is the friction force exerted on phase $i$ by viscous stresses within that phase. $\mu_{\mathrm{eff}, i}\left(\mu_{\mathrm{cfr}, i}=\mu_{i}+\mu_{t, i}\right)$ is the effective viscosity for phase $i . \quad I_{i l}$ : the momentum transfer to the phase $i$ resulting from interactions with other phases in the same control volume. Also,

$$
B_{i l}=\vec{l} \cdot R_{i} o_{i} \vec{g}
$$

The turbulence in the liquid phase was calculated by using the standard two-equation $k-\varepsilon$ model. ${ }^{22}$ ) The role of the second phase on the liquid turbulence was not modeled explicitly. The values of the empirical constants were assumed to be the same as in single phase flows. The effects of the gas phase turbulence were found to be insignificant in the process. Results were obtained by setting the gas phase turbulent viscosity to be equal to zero and 300 times the molecular gas viscosity. There was little difference in the two sets of predictions as shown in a later section. However, the results presented in this paper are for a constant gas phase effective viscosity of 300 times the molecular gas viscosity.

\subsubsection{Energy Equation}

The first law of thermodynamics implies:

$$
\begin{aligned}
& \frac{\partial}{\partial t}\left(R_{i} \rho_{i} h_{i}\right)+\operatorname{div}\left(R_{i} \rho_{i} \vec{V}_{i} h_{i}\right) \\
& \quad=\operatorname{div}\left(R_{i} \Gamma_{\text {eff }, i} \operatorname{grad} h_{i}\right)+J_{i}
\end{aligned}
$$

where, $h_{i}$ : enthalpy of the phase $i$ per unit mass. The first term on the right side represents the heat diffusion in the same phase due to the temperature gradient. $\Gamma_{\mathrm{eff}, i}$ is the effective thermal conductivity of the phase $i$, i.e.,

$$
\Gamma_{\mathrm{eff}, i}=\frac{\mu_{i}}{\sigma_{i}}+\frac{\mu_{t}}{\sigma_{t, i}}
$$

where, $\sigma_{i}, \sigma_{t, i}$ : the laminar and turbulent Prandt numbers of the phase $i$, respectively.

In the present study, $\sigma_{t, i}$ was assumed to be one for both the gas liquid phases. $J_{i}$ represents the energy exchange between the phases. $J_{i}$ is calculated as

$$
J_{i}=\alpha\left(T_{j}-T_{i}\right)
$$

where $\alpha$ represents the inter-phase heat transfer coefficient per unit volume, and subscript $j$ is like $i$, an index of the phase number. Heat generation due to the viscous dissipation was neglected in the computations. 


\subsection{Interphase Exchange Coeffcients}

In accordance with the phase momentum equations given in Eq. (3), the total force exerted by one phase on the other, in $l$-direction, can be written for unit volume as

$$
I_{i l}=C_{f}\left(u_{j l}-u_{i l}\right)
$$

here, $C_{f}$ : inter-phase friction coefficient.

The interphase friction force can also be written $\mathrm{as}^{23)}$

$$
I_{i l}=\frac{C_{D} \frac{1}{2}\left|\vec{V}_{r}\right| A_{d} R_{d} \rho_{e}}{B_{d}}\left(u_{j l}-u_{i l}\right)
$$

where, $A_{d}$ : the projected area of a typical bubble or droplet

$B_{d}$ : the volume of a typical bubble or droplet

$C_{D}$ : the dimensionless drag coefficient

$R_{d l}$ : the volume fraction of the dispersed phase.

Subscripts $c$ and $d$ are used to denote continuous and dispersed phases, respectively. Then, $C_{f}$ can be written as

$$
C_{f}=\frac{C_{D} \frac{1}{2}\left|\vec{V}_{r}\right| \rho_{c} A_{d} R_{d}}{B_{d}}
$$

The drag coefficient $C_{D}$ is an empirically or semiempirically determined parameter, which differs from one flow regime to another. In gas injection systems the gas concentration may change considerably over the flow domain, and in turn the flow regime changes. In general a highly concentrated gas region about the axis of the injection nozzle and a relatively lower gas concentration region away from the injection axis are observed. Near the injection port and in the top gas region (between $z=H_{i}$ and $z=H$ in Figs. 1 and 2) a drop-gas flow regime prevails. In the bath, the highly concentrated gas region may be considered as a churn-turbulent flow, while the region far from the axis can be classified as a bubbly flow. The churnturbulent regime is characterized by high values of relative velocity, $V_{r}$, and transition from uniform bubbling to turbulent bubbling. Formally a 0.3 gas volume fraction can be used as a criteria to distinguish between these two flow regimes as suggested by Ishii and Zuber. ${ }^{23)}$

In the churn-turbulent flow regime, the drag coefficient can be calculated as ${ }^{23)}$

$$
C_{D}=\frac{8}{3}\left(1-R_{d}\right)^{2}
$$

In the bubbly and droplet flow regions, $C_{D}$ is calculated as $^{24)}$

$$
C_{D}=\frac{24\left(1+0.15 R e_{b}^{0.687}\right)}{R e_{b}}+\frac{0.42}{1+\frac{4.25 \times 10^{4}}{R e_{b}^{1.16}}}
$$

where, $R e_{b}$ : the Reynolds number based on the bubble/drop diameter, the local continuous phase properties, and slip velocity.

In the literature, data on the size of bubbles formed at a nozzle can be found. However, bubble size in a recirculating turbulent bath would have a dynamic size distribution due the disintegration and coalescence. In the systems under consideration, the average bubble and droplet sizes were assumed to be 10 and $3 \mathrm{~mm}$, respectively, over the entire flow domain.

If inter-phase heat transfer coefficient and the local temperature difference between the phases are known, the heat exchange between the phases can be calculated. The heat transfer coefficient per unit volume and per unit temperature difference can be written as

$$
\alpha=\frac{s A_{s} R_{d}}{B_{d}}
$$

where, $A_{s}:$ surface area of a bubble or droplet

$s$ : the inter-phase heat transfer coefficient.

The heat transfer coefficients were calculated from the semiempirical expression, ${ }^{25)}$

$$
\mathcal{N} u=\frac{s d}{k_{c}}=2.0+0.6 \operatorname{Re}_{b}^{1 / 2} \operatorname{Pr}^{1 / 3}
$$

where, $d:$ the bubble diameter

$k_{c}$ : the thermal conductivity of the continuous phase

$R e_{b}:$ the Reynolds number based on the bubble/drop diameter, continuous phase properties, and the slip velocity.

The same relation was used for the entire flow domain. The bubble and droplet sizes are the same as those used in the calculations of the friction coefficients.

\subsection{Initial and Boundary Conditions}

In practical gas injection systems, the location and shape of the liquid 'free surface' does not remain sharp as the erupting gas column breaks up the liquid into droplets. Soon after the gas jet is turned on, the 'free surface' region becomes a two-phase zone characterized by high gradients of the liquid/gas volume fraction. In the present study, the computational domain was extended beyond the initial height of the undisturbed liquid level (see Figs. 1 and 2). Since the 'free surface' region is dependent on the geometry and the gas injection conditions, it is a natural outcome of the solution (flow structure in the bath) to the problem. Results presented in later sections will further clarify the point. The region close to the undisturbed free surface $\left(z=H_{i}\right)$ was considered to be within the computational domain and the nodal points in that region were considered as regular internal grid points. An advantage of the present formulation is that reasonable estimates of the location and shape of the 'free surface' region can be obtained without specifying additional conditions at this region.

The following initial and boundary conditions were used in the present analysis (see Figs. 1 and 2).

Initially, the bath was considered to be partially filled with quiescent liquid up to a height $H_{i}$ (as shown in Figs. 1 and 2). The space above the initial undisturbed liquid height was occupied by the gas 
only. Specifically:

$$
\begin{aligned}
& R_{1} \approx 0 \text { for } z \leq H_{i} \text { and } R_{1} \approx 1 \text { for } z>H_{i} \\
& R_{2} \approx 1 \text { for } z \leq H_{i} \text { and } R_{2} \approx 0 \text { for } z>H_{i}
\end{aligned}
$$

i) A symmetry axis (in the vertical injection system), and a symmetry plane (in the horizontal injection system) exist:

Symmetry conditions apply for all the variables at these boundaries.

ii) Along the vessel walls:

On the walls, the velocities satisfy the no slip condition. The logarithmic law of the wall is used to calculate the values of $k$ and $\varepsilon$ at the near wall points. It is assumed that there is no heat loss through the walls.

\section{iii) At the nozzle exit:}

Uniform velocity and temperature profiles were assumed for the gas at the injection nozzle exit. At the nozzle exit,

$$
R_{1} \approx 1 \text { and } R_{2} \approx 0
$$

The gas phase turbulence was not modeled explicitly. A zero gradient boundary condition was assigned for $k$ and $\varepsilon$ for the calculation of the liquid phase turbulence.

iv) At the top surface ( $z=H$ in Figs. 1 and 2):

In gas injection systems, the location and shape of the liquid 'free surface' are outcomes of the flow field in the vessel. Due to the gas hold up (in other words accumulation of the gas in the bath), the liquid free surface rises beyond its initial level, $H_{i}$. In order to realistically model the volume expansion due to gas injection, the computational domain was extended beyond the initial height of the liquid bath, $H_{i}$ to the height $H$.

At the top surface $(z=H)$ the gas was allowed to leave the bath at the rate it arrives at the surface. This condition was satisfied by fixing the pressure (atmospheric) at the surface. The liquid was not allowed to leave the system, and the zero shear condition was applied for both phases. Zero gradient boundary conditions for $k$ and $\varepsilon$ wcre introduced at the top. The temperature boundary condition at the top surface for the gas phase was satisfied by allowing the heat leave with the gas. Zero gradient boundary conditions were also assumed for the temperatures of the both phases.

\section{Numerical Solution}

The differential equations were discretized using a control volume based method over a staggered grid system. A hybrid differencing scheme was cmployed to approximate the convection and diffusion terms. A fully implicit differencing scheme was applied for transient terms. Time-steps of $5 \mathrm{~ms}$ were used in the computations. The resulting systems of the equations were solved using the general purpose computer program PHOENICS. ${ }^{26}$ ) The time evolution of the velocity, pressure, volume fraction and the temperature fields were solved for both the gas and liquid phases.

\section{Results and Discussion}

Two industrial scale baths, one with vertical and the other with horizontal injection were considered. In these systems, a stream of gas was injected into the molten iron. The vertical injection case was assumed to be isothermal, i.e., the gas temperature at the nozzle exit and the molten iron temperatures are the same. The velocity and volume fraction fields were predicted for the gas and liquid phases. In the horizontal injection case, a non-isothermal system was considered. For this system, the temperature fields were also predicted for both phases in addition to the velocity and volume fraction fields.

\subsection{Vertical Injection}

A cylindrical vessel (Fig. 1) of $1.06 \mathrm{~m}$ diameter was considered. The vessel contained molten iron, at $1873 \mathrm{~K}$, filled up to $0.609 \mathrm{~m}$ from the bottom. A stream of air was injected vertically through an annular nozzle located centrally at the bottom of the tank. The inside and outside diameters of the inner tube are 0.005 and $0.01 \mathrm{~m}$, respectively. The inside diameter of the outer tube is $0.015 \mathrm{~m}$. The gas was injected with a uniform velocity of $100 \mathrm{~m} / \mathrm{s}$ through both tubes. The flow was assumed to be axisymmetric. Computations were performed in transient mode. A mesh system of $25 \times 26(r \times z)$ was used. After preliminary calculations this mesh system was found to be a good compromise between the computational accuracy and cost.

The pressure at the exit of the nozzle was assumed to be the same as the hydrostatic pressure. It was assumed that the air reaches the liquid temperature immediately upon injection. Thus, the air properties at the nozzle exit were calculated considering the hydrostatic pressure of the liquid and the temperature of the bath. All the fluid properties were assumed to be constant over the flow domain. The results presented here give important insights to flows induced by the gas injected due to hydrodynamic effects only.

Fig. 3 shows the time variations of the gas volume fraction and the velocity at a point $(r=0.01 \mathrm{~m}, z=$ $0.28 \mathrm{~m})$. As expected a quasi-steady condition is reached after a highly oscillatory state. These violent oscillations are the initial transients. Computations were performed for $0.6 \mathrm{~s}$. By this time there were no considerable changes in the flow field, thus the calculations were terminated.

In the gas injected baths, the distribution of the gas volume fraction is an important parameter to characterize the system. It is the concentration fields of the phases that control the interaction between the phases. The gas volume fraction maps at 0.32 and $0.6 \mathrm{~s}$ are given in Figs. 4 and 5, respectively. These figures show that the gas concentration around the axis of the injection nozzle is quite high. However, its radial dispersion is constrained within a cone of a very small angle. An estimate of the liquid 'free sur- 


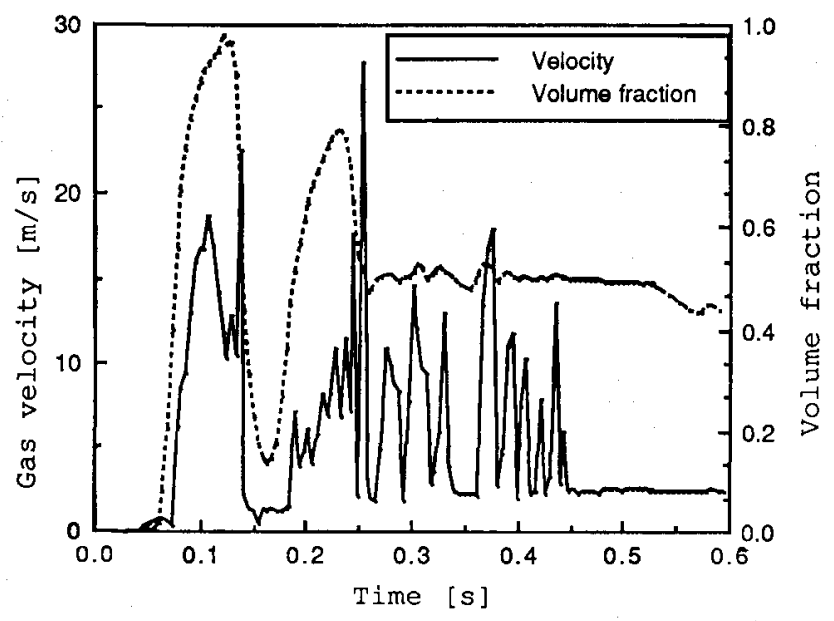

Fig. 3. Time variations of the gas volume fraction and velocity at point $(r=0.01 \mathrm{~m}, z=0.28 \mathrm{~m})$.

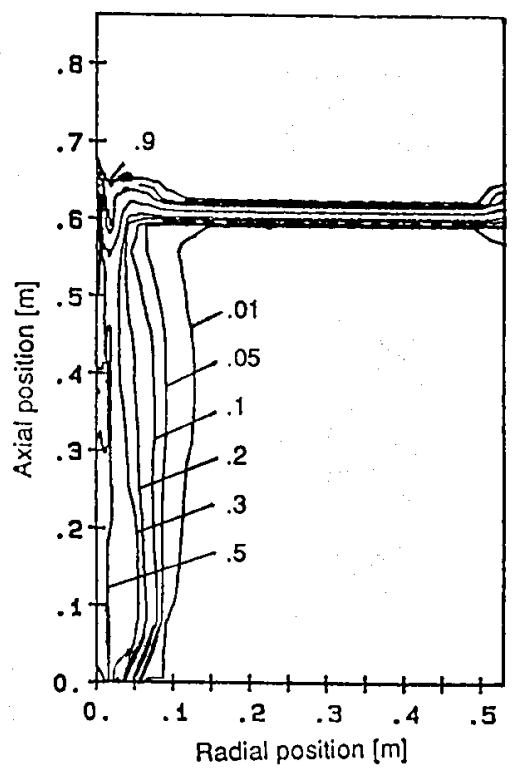

Fig. 4. Gas volume fraction contours at time $0.32 \mathrm{~s}$ after injection started.

face' can also be obtained from these figures (as characterized by the region of high gradient of the volume fraction contours). Fig. 5 shows that the liquid 'free surface' is not flat. This is also indicative of considerable liquid splashing due to the gas injection.

The axial components of the gas and liquid velocities (at $0.6 \mathrm{~s}$ ) are plotted in Fig. 6. As seen in this figure, the gas velocity decreases in a short distance from the injection nozzle then the gas attains a relatively uniform velocity. This sugests that the gas loses its kinetic energy around the nozzle; thereafter buoyancy dominates the flow. Several investigators have also observed similar trends in theoretical and experimental studies. ${ }^{1-3}$ ) Thus, it can be concluded that the mixing in the vessel mainly depends on the gas flow rate rather than on gas inlet velocity. Our calculations carried out on air-water systems to investigate the effects of the gas mass flow rate in the liquid circulation rate also supports this argument.

As discussed earlier, in the present computations, the liquid phase turbulence viscosity was solved as a

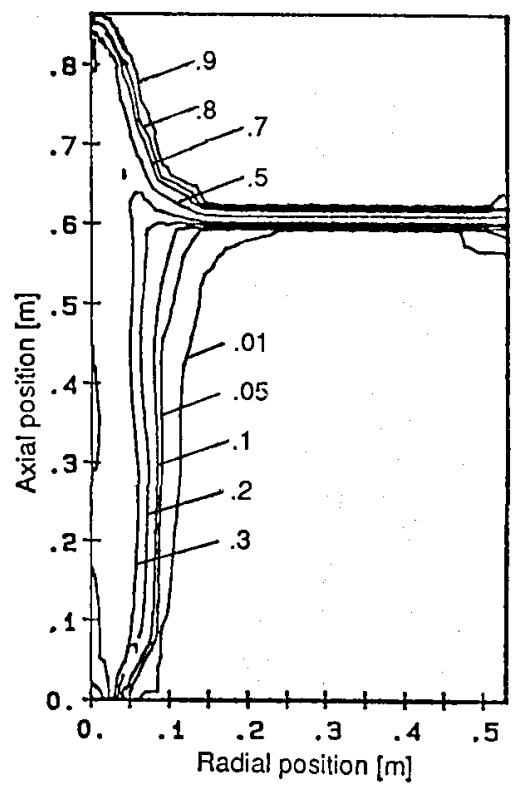

Fig. 5. Gas volume fraction contours at time $0.6 \mathrm{~s}$ after injection started.

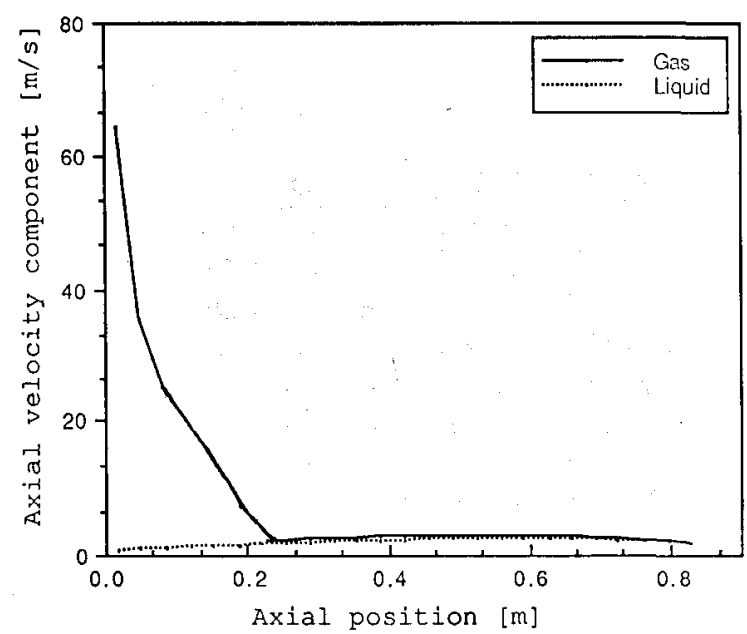

Fig. 6. Axial velocity profiles of the gas and liquid phases along the center line.

field variable by using the $k-\varepsilon$ two-equation model. The axial profiles of the gas velocities obtained with zero gas phase turbulence and with 300 times the molecular viscosity are compared in Fig. 7. As seen, the gas phase turbulence has little effect on the results. However, the computations with zero gas phase turbulence voscosity predicted slightly higher gas velocities in the regions where the gas concentration is relatively higher.

\subsection{Horizontal Injection}

A cubical vessel with square cross section $(0.74 \mathrm{~m}$ $\times 0.74 \mathrm{~m}$ ) was considered for the horizontal injection study (Fig. 2). The height of the vessel is $0.75 \mathrm{~m}$. The vessel contained molten iron up to a height of $0.49 \mathrm{~m}$ from the bottom. The initial temperature of the melt was $1873 \mathrm{~K}$. A stream of hot gas mixture at $2473 \mathrm{~K}$ was injected horizontally through a nozzle. For the calculations the gas mixture properties were approximated by air properties. The nozzle is lo- 
cated $0.18 \mathrm{~m}$ above the bottom of the vessel and centrally in the horizontal direction on one of the side walls. The nozzle cross-sectional area is $33.16 \mathrm{~cm}^{2}$. The gas was injected with a horizontal velocity of $250 \mathrm{~m} / \mathrm{s}$. Gas compressibility was explicitly considered in the model. The gas density was calculated using the ideal gas law. Since the vertical plane passing through the nozzle axis is a symmetry plane, computations were performed in the half domain using a $10 \times$ $17 \times 17(x, y, z)$ mesh system.

Fig. 8 shows the gas volume fraction map on the vertical plane passing through the nozzle axis, $0.15 \mathrm{~s}$ after injection started. The location of the nozzle is shown by an arrow in the figure. As seen, the injected gas rises along the wall on which the nozzle is located. Around the injection nozzle, there is a gas rich region. The gas penetration in the injection direction $(y)$ is small. This indicates that the gas loses its kinetic energy in a short distance from the

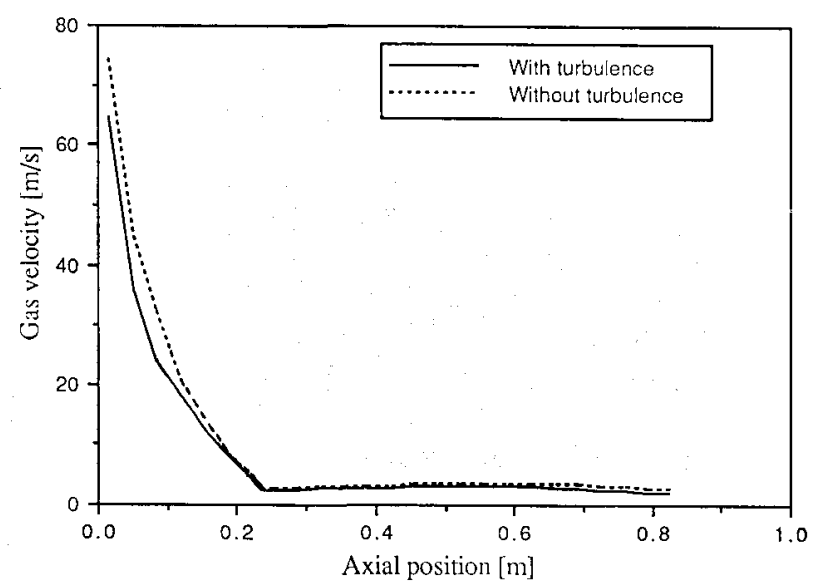

Fig. 7. The velocity profiles along the axis of the injected nozzle predicted with and without gas turbulence.

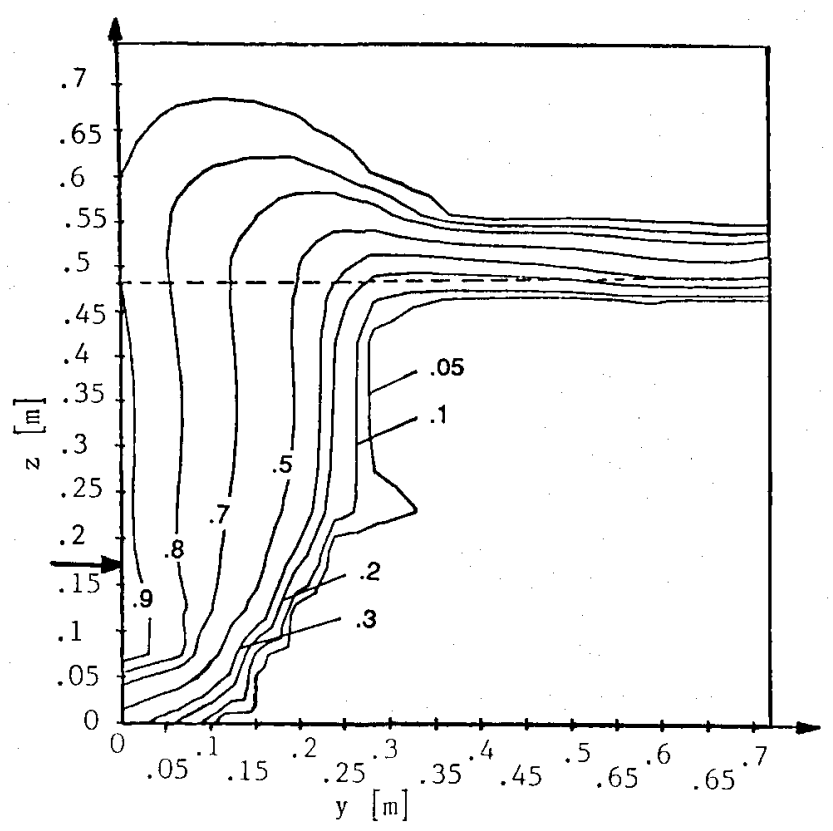

Fig. 8. Gas volume fraction contrours for the horizontal injection system on the vertical plane passing through the nozzle axis, at $0.15 \mathrm{~s}$. nozzle exit and then it starts rising vertically due to the buoyancy force. The gas volume fraction map at the horizontal plane passing through the nozzle axis is shown in Fig. 9. Due to the symmetry conditions, only one half of the plane is shown. The penetration of the gas plume in this plane is considerably less due to the absence of buoyancy effects. This result again confirms the earlier observation that the kinetic energy of the gas jet alone has little effect on the gas plume development.

Along the axis of the injection nozzle, the $y$ and $z$ components of the velocity vectors, for the gas and liquid phases are plotted in Figs. 10 and 11 , respectively. Fig. 10 shows that the $y$ component of the gas velocity decreases drastically within a short distance from the nozzle exit. The $z$ (vertical) component of the gas velocity (as shown in Fig. 11) is initially zero at the nozzle exit. Then, it reaches a maximum value and drops off. In Fig. 12, the $x$ component of the gas and liquid velocities along the line parallel to the $y$ axis at the horizontal plane passing through the nozzle axis (refer to Fig. 2) are plotted. It is seen from this figure that the flow close to the injection location is outward from the nozzle axis, while it is toward the nozzle axis near the opposite wall. These predicted results are in agreement with physical expectations.

\subsection{Heat Transfer Predictions}

The gas was injected at $2473 \mathrm{~K}$ with the velocity $250 \mathrm{~m} / \mathrm{s}$. Initial temperature of the molten iron was assumed to be $1873 \mathrm{~K}$. At the nozzle exit the gas pressure was assumed to be the same as the hydrostatic pressure. Calculations showed that the heat transfer between the gas and the liquid is very efficient. The gas leaving the nozzle assumes the bath temperature in a short distance from the injection nozzle. The gas temperature field on a vertical plane passing through the axis of the injection nozzle, at $0.15 \mathrm{~s}$ after injection, is given in Fig. 13. The temperature field of the gas is different from the temperature field of the liquid only around the injection nozzle where the gas concentration is very high. Thereafter there is no difference between the gas and liquid temperatures. The overall liquid temperature has not changed much from its initial temperature. This is mainly due to

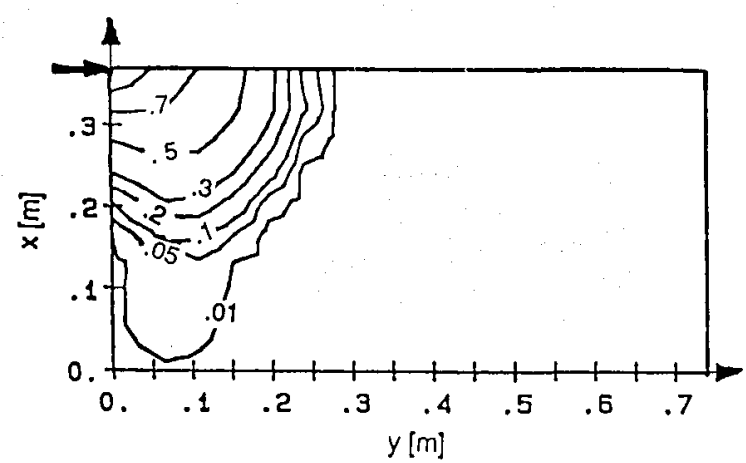

Fig. 9. Gas volume fraction contours for the horizontal injection system on the horizontal plane passing through the nozzle axis, at $0.15 \mathrm{~s}$. 


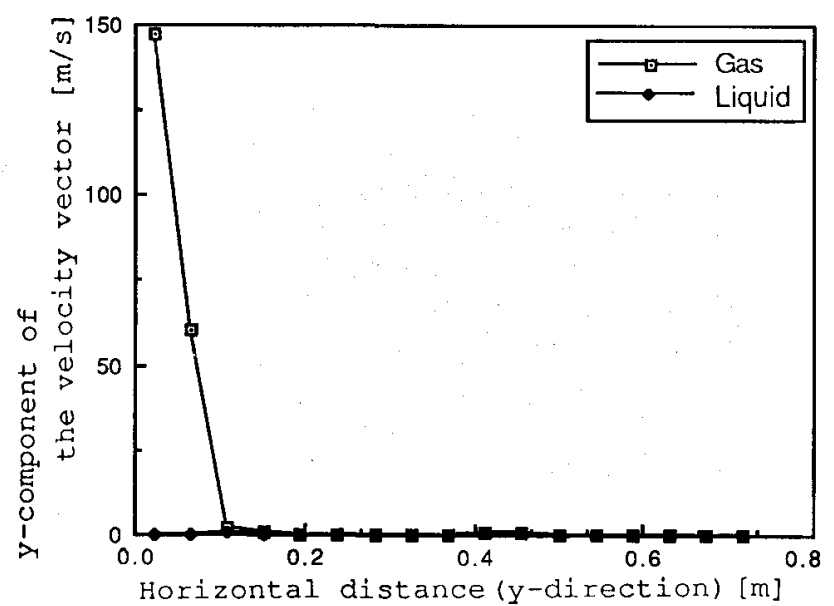

Fig. 10. Profiles of the $y$-component of the gas and liquid velocities along the axis of injection nozzle, at $0.15 \mathrm{~s}$.

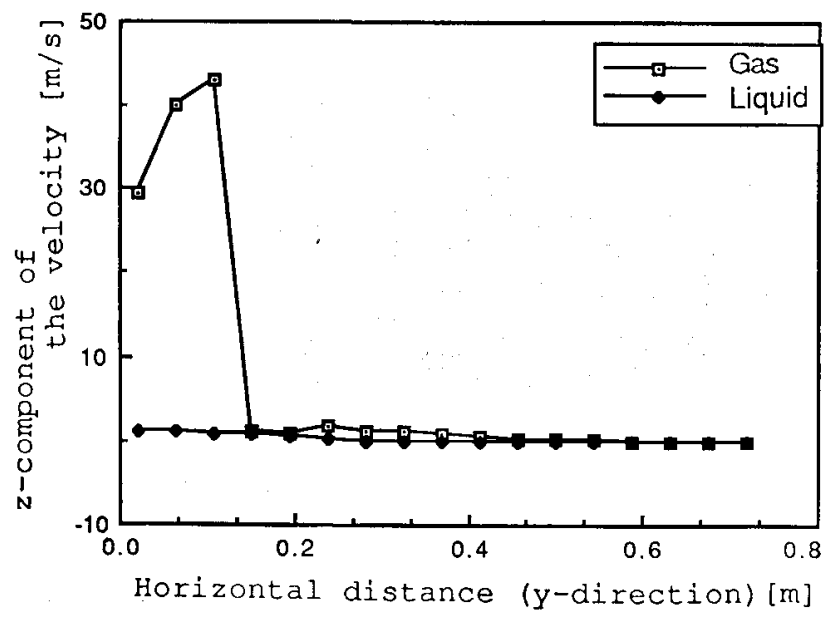

Fig. 11. Profiles of the $z$-component of the gas and liquid velocities along the injection axis, at $0.15 \mathrm{~s}$.

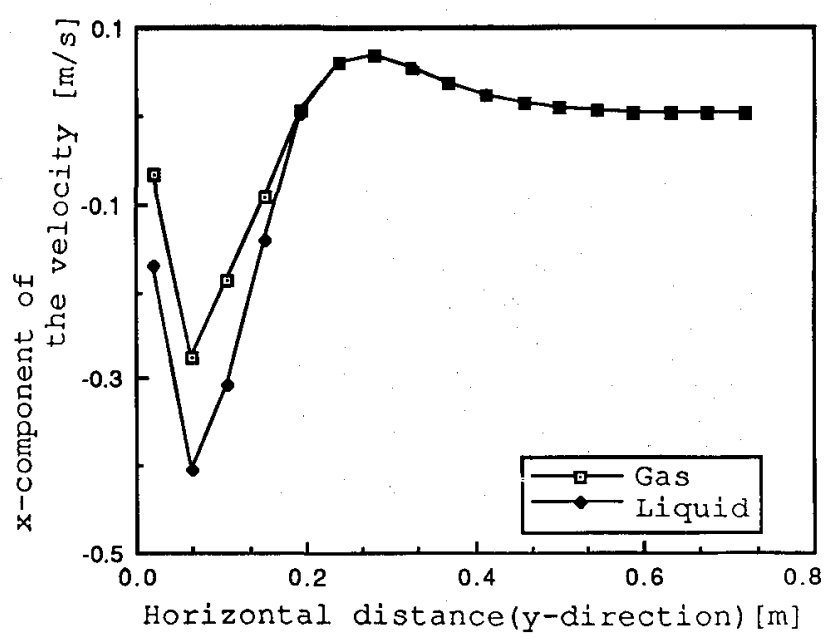

Fig. 12. Profiles of the $x$-component of the gas and liquid velocities along the line parallel to the nozzle exit on the horizontal plane passing through the injection nozzle, at $0.15 \mathrm{~s}$.

the very short elapsed time $(0.15 \mathrm{~s})$ considered in the calculations and the large difference in thermal capacities of the liquid and gas phases.

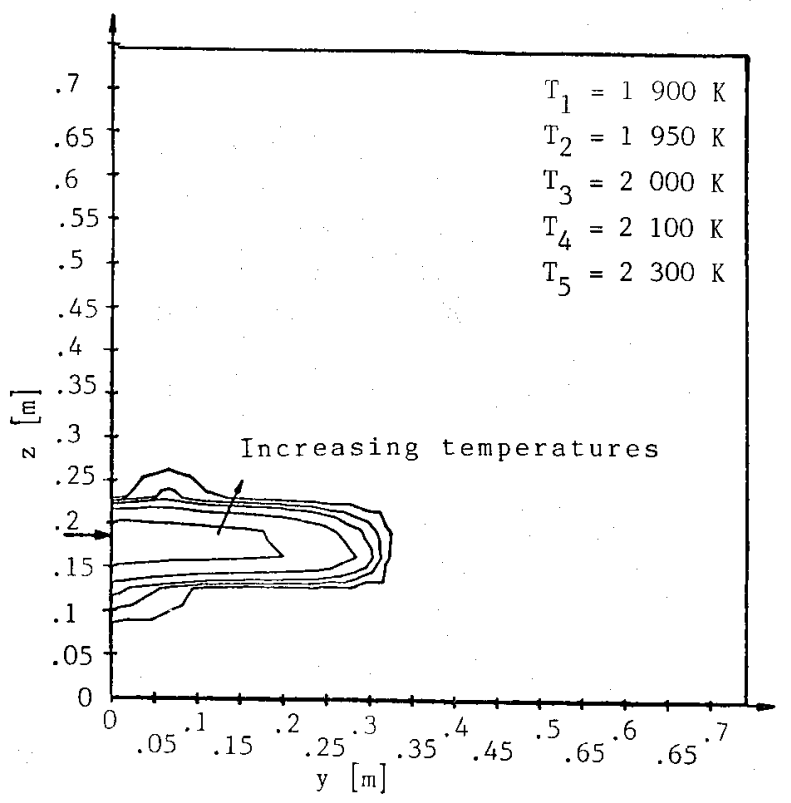

Fig. 13. Temperature contours of the gas phase for the horizontal injection case, at $0.15 \mathrm{~s}$.

\section{Conclusions}

A two-phase time-dependent three-dimensional numerical model has been developed to predict the transport processes in gas injected molten iron baths. Due to harsh conditions and high cost of experimental studies, reliable numerical models are needed for the prediction of transport processes (including mixing) in metallurgical operations and other practical gas injected liquid baths. The velocity and the temperature fields for both phases were predicted in a cylindrical molten iron bath due to the vertical injection of the air, and in a cubical molten iron bath due to the horizontal injection. The Navier-Stokes and the energy equations were solved for each phase. Interaction of the phases with each other were represented through the interphase exchange coefficients. The turbulence for the liquid phase was modeled by using the two-equation $k-\varepsilon$ model. The gas phase was assigned an effective viscosity.

The flow processes in gas injected molten iron baths are complex and several subareas (e.g., inter-phase momentum and heat transfer, turbulence, dispersion of phases, etc.) need to be addressed for realistic modeling efforts. The present work extends past research efforts in several of the above areas.

An interesting feature of the present work is the way the 'free surface' region is modeled. Unlike other studies reported in the literature, we extended the computational domain beyond the initial height of the liquid. In most past studies, the computational domain is identical to the volume occupied by the undisturbed liquid. A zero axial liquid velocity is assumed as a boundary condition at the initial free surface. The above assumptions may be reasonable only for very low injection rates and are unacceptable for practical gas injected liquid baths. In the present work, the top boundary of the computational domain is considered well above the initial height of the liquid 
so that the penetrating gas column and the shape of the 'free surface' region can be predicted in a realistic way. This approach also provides an effective way to incorporate the volume change in the bath due to the accumulation of the gas in the liquid.

The heat transfer between the two phases is of critical importance in some processes (particularly steelmaking). The effect of volume changes in the gas due to heat transfer was considered explicitly in the model. The present model is thus capable of predicting the time evolution of the velocity, volume fraction and temperature fields of individual phases within the gas injected liquid bath. When a gas stream with a higher temperature is injected in a liquid bath (at a lower temperature), most of the heat exchange between the phases is found to take place in a small region around the injection nozzle. Since the heat capacities of the gas and the liquid are vastly different (for the system considered), a significant amount of gas flow will be required to achieve any appreciable effect on the liquid temperature. For any quantitative predictions in metallurgical systems, the present model is expected to provide useful information regarding heat transfer.

The computations revealed that two main flow regimes exist in the plume for both vertical and horizontal injection. In a small region close to the nozzle exit, the flow field is strongly affected by the kinetic energy of the gas. Further from the nozzle is the buoyancy dominated region, where the flow parameters are relatively uniform. In the horizontal injection system, the gas penetrates into a short distance in the horizontal direction, then it rises vertically up due to the buoyancy. Thus it can be concluded that the buoyancy is the main force driving the flow in the vessel.

Effect of gas phase turbulence on the flow predictions was evaluated. The predictions with and without the turbulent gas viscosity showed that the gas turbulence has a small role on the flow field. This can provide significant savings in computational time in the modeling of gas injected liquid baths.

\section{Acknowledgment}

This study was supported in part by the Commonwealth of Pennsylvania's Ben Franklin Partnership through the Advanced Technology Center of Southeastern Pennsylvania and Air Products and Chemicals, Inc.

\section{Nomenclature}

$A$ : projected area of a bubble or droplet

$A_{s}$ : bubble/droplet surface area

$B$ : volume of a bubble or droplet

$C_{D}$ : dimensionless drag coefficient

$C_{f}$ : interphase friction coefficient for unit volume and unit relative velocity

$d$ : bubble/droplet diameter

$D_{t}$ : phase dispersion coefficient

$g$ : gravitational acceleration

$h$ : gas/liquid enthalpy

$H_{i}$ : initial liquid height
$H$ : height of the computational domain

$k$ : kinetic energy of turbulence

$\mathcal{N u}:$ Nusselt number

$P$ : pressure

$R$ : phase volume fraction

$R e_{b}$ : bubble Reynolds number

$R e$ : jet Reynolds number (based on orifice diameter)

$s$ : bubble/drop heat transfer coefficient

$t$ : time

$T:$ phase temperature

$V_{r}$ : relative velocity vector

$\alpha$ : interphase heat transfer coefficient

$\Gamma_{\text {eff }}$ : effective heat diffusion coefficient, $\left(\Gamma_{\text {eff }}=\Gamma+\right.$ $\left.\Gamma_{1}\right)$

$\varepsilon$ : dissipation rate of turbulence kinetic energy

$\mu:$ molecular viscosity

$\mu_{\mathrm{eff}}:$ effective viscosity, $\left(\mu_{\mathrm{eff}}=\mu+\mu_{t}\right)$

$\mu_{t}:$ turbulent viscosity

$\rho:$ density

$\sigma$ : Prandtl number

$\sigma_{d}:$ dispersion Prandtl number, $\sigma_{d}=\nu_{t} / D_{t}$

$\sigma_{t}:$ turbulent Prandtl number, $\left(\sigma_{t}=\nu_{t} / \Gamma_{t}\right)$

Subscripts

1: gas phase

2: liquid phase

$c$ : continuous phase

$d$ : dispersed phase

$i$ : phase indices

\section{REFERENCES}

1) A. H. Castillejos and J. K. Brimacombe: Metall. Trans. B., 18B (1987), 659 .

2) H. Turkoglu and B. Farouk: to be published in Metall. Trans. B, (1990).

3) J. Szekely, H. J. Wang and K. M. Kisser: Metall. Trans. B, 7B (1976), 287.

4) M. Sano and K. Mori: Trans. Iron Steel Inst. Jpn., 23, (1983), 169.

5) N. El-Kaddah and J. Szekely: Ironmaking Steelmaking, 8 (1981), 269.

6) J. H. Grevet, J. Szekely and N. El-Kaddah: Int. J. Heat and Mass Transfer, 25 (1982), 487.

7) T. Deb Roy, A. K. Majumadar and D. B. Spalding: Appl. Math. Mod., 2 (1978), 146.

8) T. Deb Roy and A. K. Majumadar: J. Met. (1981), Nov., 42.

9) Y. Sahai and R.I.L. Guthrie: Metall. Trans. B, 13B (1982), 203.

10) D. Mazumdar, and R.I.L. Guthrie: Metall. Trans. B, 16B (1985), 83

11) M. Salcudean, K.Y.M. Lai and R.I.L. Guthrie: Can. J. Chem. Eng., 63 (1985), 51.

12) M. Salcudean and K.Y.M. Lai: Numer. Heat Transfer, 14 (1988), 97.

13) G. Oryall and J. K. Brimacombe: Metall. Trans. B, 7B (1976), 391.

14) E. O. Hoefele and J. K. Brimacombe: Metall. Trans. B, 10B (1979), 631.

15) J. W. McKelliget, M. Cross and R. D. Gibson: Appl. Math. Mod., 6 (1982), 469.

16) M. Cross and N. G. Markatos: ISS Proc. of the 4th Process Technology Conference: Mixed Gas Blowing, Vol. 4, (1984), 11. 
17) C Aldham, M. Cross and N. C. Markatos: Proc. N. E. Polytechnics Mathematical Modeling and Computer Simulation Group, P. G. Hudson, ed., EMJOC Press, London, (1982), 65.

18) M. Cross and N. C. Markatos: Soc. Min. Eng., (1984), 291.

19) P.L.T. Koh, N. C. Markatos and M. Cross: PhyscoChemical Hydrodydamics, 9 (1987), 197.

20) M. P. Schwarz, J. K. Wright and B. R. Baldock: The Mathematical Modeling of Materials Proceesing, J. Szekely, ed., Miner. Met. Mater. Soc., Warrendale, PA, (1987).

21) D. B. Spalding: Recent Advances in Numerical Methods in Fluids, C. Taylor, ed., Pinebridge Press, London, (1980), 139.

22) B. E. Launder and D. B. Spalding: Mathematical Models of Turbulence, Academic Press, New York, (1982).

23) M. Ishii and N. Zuber: AIChE J., 25 (1979), 843.

24) R. Cliff, J. R. Grace and M. E. Weber: Bubbles, Drops and Particles, Academic Press Inc., New York, (1978).

25) W. E. Ranz and W. R. Marshall: Chem. Eng. Prog., 48 (1952), 141 and 173 .

26) D. B. Spalding: Mathem. Computers in Simulation, 13 (1981), 267. 